\title{
A Variable Step-Size LMS Adaptive Filtering Algorithm Based on Error Feedback
}

\author{
Fengchun Wang ${ }^{1, a}$, Jiyan $\mathrm{Du}^{1, \mathrm{~b}}$ \\ ${ }^{1}$ Institute of UAV Army Officer Academy, Hefei 230000, China \\ aemail: yumao0916@sina.com, bemail:zhang_zdc@163.com
}

Keywords:LMS Algorithm; Adaptive Filtering; Convergence Rate; Steady-state Error

\begin{abstract}
In order to solve the problem of LMS algorithm, a new variable step-size LMS algorithm is studied. The algorithm is based on the sigmoid function which builds the non-linear functional relationship between step and error. By introducing the error feedback strategy to adjust the parameters adaptively, the algorithm solves the problem of setting parameters in the function. Compared with other algorithms, simulation results show that the algorithm performs perfect at convergence rate and steady-state error with a better applicability.
\end{abstract}

\section{Introduction}

The algorithm of LMS (Least mean square)[1] is widely used in many areas as radar system due to its simpleness. LMS algorithm is based on the steepest descent principle; it replaced the random grad by instant grad and reach at the optimized weight along the negative direction of weight grad to minimize the mean square error. But the requirement for the convergence rate and the steadystate error by LMS algorithm with the fixed step size is contradictory. Reference [2] presented the algorithm that variable step-size parameter is proportional to the error, as improves the performance, but not ideal. Reference [3] presented a method called S function variable step-size LMS algorithm (SVLMS), step adjustment strategy of the algorithm is advanced to some extent, but the step changes sharply when the algorithm error approximate zero, as may increase the steady-state error. Reference [4] made further improvement on the basis of reference [3], and corrected the step sharp changes when the algorithm error approximate zero, but the key parameters in the algorithms need to set manually, and the improper parameter setting will seriously affect the performance of the proposed algorithm. Reference [5-6] presented the relevant variable step-size LMS algorithm respectively based on versiera, hyperbolic tangent function and arctan function to remit the contradiction between convergence rate and steady-state error partly.

This paper presents the Error Feedback Least Mean Square (EFLMS) algorithm based on Sigmoid function referring to Reference [4].The algorithm uses the feedback idea by introducing the parameter error factor to solve problems on Sigmoid parameter setting, it can improve the convergence rate, steady-state error and other performance indexes, and it has the extensive adaptability.

\section{Related Work}

The essence of variable step-size LMS filtering algorithm is to regulate the step size through the error adapting. Reference [4] presented a variable step-size algorithm based on sigmoid function, by which the higher convergence rate and less steady-state error could be achieved meanwhile. The sigmoid function of the algorithm is relatively simple and change slowly when the error approximate zero. The algorithm design as following:

$$
\begin{aligned}
& \mathrm{e}(\mathrm{n})=\mathrm{d}(\mathrm{n})-\mathrm{X}^{\mathrm{T}}(\mathrm{n}) \times \mathrm{W}(\mathrm{n}) \\
& \mu(\mathrm{n})=\beta\left(1-\exp \left(-\alpha|\mathrm{e}(\mathrm{n})|^{2}\right)\right) \\
& \mathrm{W}(\mathrm{n}+1)=W(\mathrm{n})+2 \mu(\mathrm{n}) \times \mathrm{e}(\mathrm{n}) \times \mathrm{X}(\mathrm{n})
\end{aligned}
$$

Where: $d(n)$-expected output value of signal; $X(n)$-input signal vector of time $n$; $W(n)$-weight vector of adaptive filter at time $n$; e(n)-error signal; $\mu$ (n)-regulated step size. 
It shows that the regulated step size of the algorithm is changing as the changing of error e(n), that is the variable step-size LMS algorithm. The core of the algorithm is formula 2, by which to set up the corresponding relationship between $\operatorname{error}\{\mathrm{e}(\mathrm{n})\}$ and $\operatorname{step}\{\mu(\mathrm{n})\}$, when step size increase as error increasing, the convergence rate can be improved; when step size decrease as error decreasing, the algorithm can tend to steady state. The $\alpha$ is one of the key parameter for algorithm performance, its value will directly affect the convergence rate and steady-state error value. But, the Reference [4] did not present clear method for parameters setting. There're different requirements on algorithm performance for different applications, it is not so easy to determine a reasonable parameter through the experiment so as to meet the application demand. The improper setting of parameter will not only seriously affect the performance of the algorithm, but also limit the applicability of the algorithm.

The proposed EFLMS (Error Feedback Least Mean Square) algorithm is based on Sigmoid function, through the introduction of parameter error factor to solve the function parameter settings problem. The description of the EFLMS algorithm is as following chapter 3.

\section{Algorithm Description And Simulation}

EFLMS algorithm is proposed based on the variable step-size strategy in Reference [4], and made a further improvement to through the feedback and achieves the parameters automatic regulating through the error introduction.

Parameter $\alpha$ in step-size function of previous algorithm is a key value for algorithm performance. it is necessary to improve the step-size function in function 2 so as to change the fixed value into function. Algorithm of design step-size and error is as follows:
$\mu$
$(n)=$
$\beta(n)(1-\exp$
$(-\alpha(n)$
$\times$
$\left.|\mathrm{e}(\mathrm{n})|^{2}\right)$
)$$
\alpha(n)=
$$
$\beta(n-1)+$$$
\mathrm{e}(\mathrm{n}) /\left.\mathrm{e}(\mathrm{n}-1)\right|^{\mathrm{k}}
$$
$\mathrm{b}_{0}[\mathrm{e}(\mathrm{n}) / \mathrm{e}(\mathrm{n}-1)]^{2}-1$

The constant $\mathrm{k}$ in formula 5 will control $\alpha(\mathrm{n})$ to regulate the convergence rate and steady-state error. The constant $0<\mathrm{b}_{0}<1$ in formula 6 will ensure the convergence. The parameter $\alpha(\mathrm{n})$ control the shape and speed of step-size variety of algorithm, but parameter $\beta(n)$ control the value range of the function.

Two parameters $\alpha(n)$ and $\beta(n)$ are the function of error ratio e(n)/e(n-1). When the ratio e(n)/e(n1) is larger, it proves the error change greatly and algorithm is still in convergence stage, then the parameter $\alpha(n)$ correspond to a large value, the algorithm convergence is accelerating. With the error ratio $\mathrm{e}(\mathrm{n}) / \mathrm{e}(\mathrm{n}-1)$ becomes smaller, the smaller $\alpha(\mathrm{n})$ result in the smaller step size $\mu(\mathrm{n})$, the algorithm tends to a steady state. So does the parameter of $\beta(n)$.

Constant $\mathrm{k}$ can be used to adjust the change speed of $\alpha(\mathrm{n})$ which will increase as the increasing of $\mathrm{k}$ value. If quick convergence is required, increase the $\mathrm{k}$ value; if higher accuracy is required, choose the smaller $k$ value. Parameter $b_{0}$ is used to ensure the convergence; different $b_{0}$ values could affect the algorithm performance.

The simulation realization of EFLMS algorithm is as following in the MATLAB simulation platform to verify the performance of the algorithm.

Experiment 1: The main performance indexes of adaptive filter algorithm are convergence rate and steady-state error. Both of EFLMS and previous algorithms choose the variable step-size strategy; it could get quick convergence and minor steady-state error through regulating the step size. The changing of step size can reflect whether the algorithm is reasonable or not. Therefore, it is necessary to do the simulation on MATLAB for step size and error to verify the performance.

The simulation settings are as follows. The input signal frequency is sine wave; referenced input signal is zero mean and variance of 1 Gauss white noise; the sampling points are set to 900.In the Reference [4], $\alpha=0.5, \beta=0.01$. And the parameter setting in EFLMS are as follows: $\mathrm{k}=2$ in Formula $5, b_{0}=0.6$ in Formula 6. The simulation results are shown in Figure 1. 

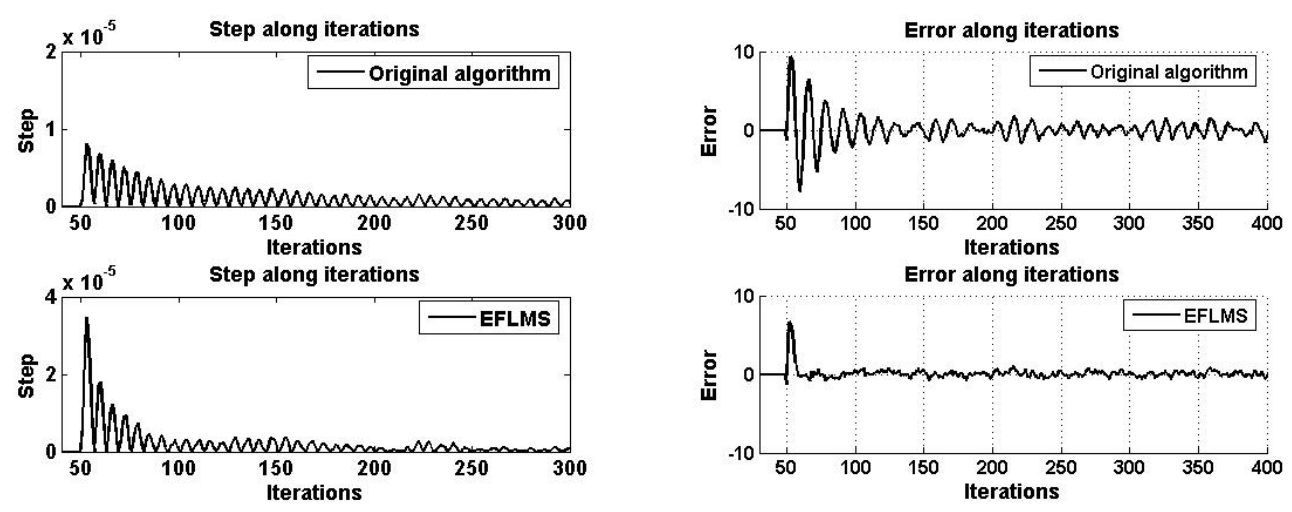

(a)Step size graph

(b) Error graph

Fig.1. The results of experiment 1

Experiment 2: Parameter $\mathrm{k}$ and $\mathrm{b}_{0}$ were used in EFLMS algorithm, as could adjust the performance of algorithm. Parameter of $\mathrm{k}$ could control the convergence rate and $\mathrm{b}_{0}$ could control convergence. For experiment 2, keep the same settings for input signal and others as experiment 1 , then fix $b_{0}$ and adjust $K$ values to check the performance change. If $b_{0}=0.4$, respectively set $k=0.5$, $\mathrm{k}=2$ and $\mathrm{k}=4$, results refer to Figure 2 (a) and Figure 2 (b).

Next, fix $k$ value and adjust $b_{0}$ in the case of convergence to check the performance change as changing of $b_{0}$. It shows that convergence doesn't occur sometimes when $b_{0}<0.2$. If $k=1$, respectively set $b_{0}=0.4$ and $b_{0}=0.9$, results refer to Figure 2 (c).

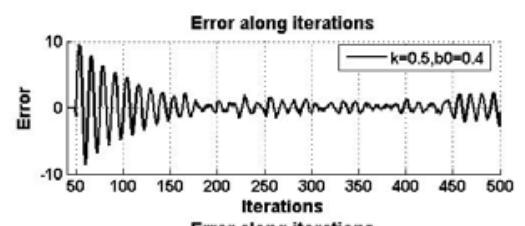

Error along iterations

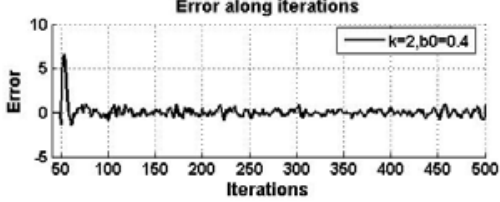

.(a)Error graph of different $\mathrm{k}$
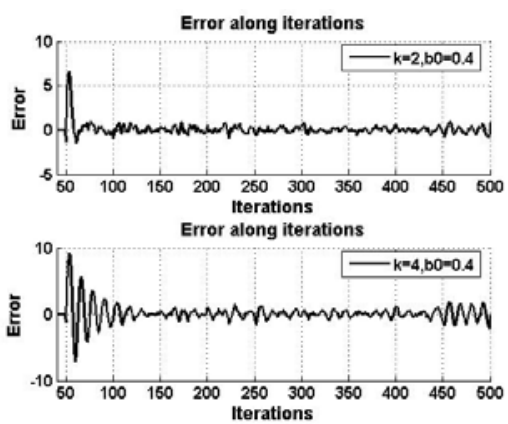

(b) Error graph of different $\mathrm{k}$
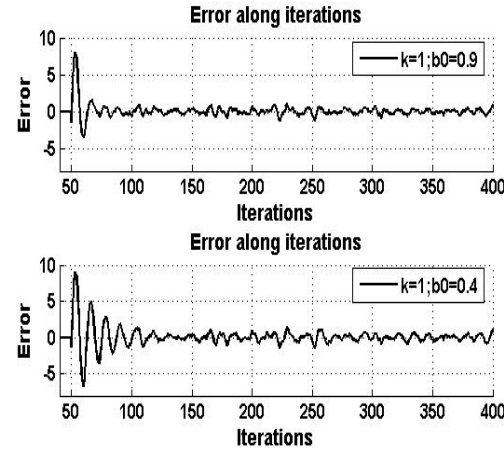

(c) Error graph of different $\mathrm{b}_{0}$

Fi.2. The results of experiment 2

\section{Simulation Analysis}

Figure 1 (a) is the changing graph of iterations of step size for both algorithms, the first curve is for EFLMS algorithm, and the other curve is for previous algorithm. When the previous algorithm gets convergence, its step size is bigger than that of EFLMS algorithm, as will influence the convergence accuracy. Furthermore, the fluctuation of step-size curve of previous algorithm is greater than that of EFLMS algorithm, which shows that its tracking speed is not as good as EFLMS algorithm. The two parameters of EFLMS algorithm are the function of $|\mathrm{e}(\mathrm{n}) / \mathrm{e}(\mathrm{n}-1)|$. Variation of step size is sensitive to error and it has better regulation ability, as will make the EFLMS algorithm converge faster with stable variation of step size and better stability.

Figure 1(b) is the error graph for both algorithms. It shows that EFLMS is faster in convergence. Fluctuations occurred for both algorithms after stabilization, as proves that there are errors, but error of EFLMS algorithm is less than original algorithm.

In figure 2 (a), $b_{0}$ value was fixed, to set $\mathrm{k}=0.5$ and $\mathrm{k}=2$ respectively. It shows that the convergence rate and steady-state error when $\mathrm{k}=2$ is less than that when $\mathrm{k}=0.5$. But, it is not so good to set rather high $\mathrm{k}$ value, because it will cause the fluctuations on step size, as could influence the convergence rate and increase error. Figure 2 (b) compares the errors when $k=2$ and $k=4$, it shows that the convergence rate and accuracy were influenced due to higher $\mathrm{k}$ value. 
The parameter of $b_{0}$ could ensure the convergence, but different $b_{0}$ values also could make influences to the algorithm. In Figure 2 (c), the first curve is the graph when $b_{0}=0.9$, and the other curve is the graph when $b_{0}=0.4$. Convergence rate increased as the increasing of $b_{0}$ values, there's less difference when stabilized for both curves, but performance is better when $b_{0}=0.9$.

\section{Conclusion}

This paper presents the variable step-size LMS adaptive filtering algorithm based on the Sigmoid function---EFLMS algorithm. It could adjust the parameters through the introduction of error factor and solve the manual setting problems for parameters in Sigmoid function, and the step-size regulating strategy could become more reasonable. The verification on simulation platform shows that the EFLMS algorithm has the higher convergence rate and higher accuracy than algorithm in Reference [4], meanwhile, the feasibility and superiority was verified.

\section{References}

[1]Widrow B,Stearns S D.”Adaptive Signal Processing”.Prentice Hall, Englewood Cliffs,NJ,1985 [2]Yasukawa H, Shimada S, Furukrawa I, et al. "Acuoustic echo canceller with high speech quality “.IEEE ICASSP, 1987:2125-2128

[3]Jingfan QIN, Jingzheng OUYANG, "A new variable step size adaptive filtering algorithm", Journal of Data Acquisition\&Processing, 1997,12(3):171-194.

[4]Ying GAO, Shengli XIE, "A variable step size LMS adaptive filtering algorithm and its analysis", Journal of Electronics,2001,29(8):1094-1097.

[5]Jingbo DENG, Xingguo HOU, "Variable step adaptive filtering LMS algorithm based on tonguelike curve", Journal of Data Acquisition\&Processing,2004,19(3):282-285.

[6]Shasha Zheng, "Research and simulation of least mean square algorithm with variable step size for smart antenna", JiLin University PhD thesis,2007. 